\title{
ÉQUIVALENCE ET NORMES EN TRADUCTION JURIDIQUE
}

\section{INTRODUCTION}

L'étude comparative de deux traductions pose nécessairement la question de l'équivalence. Aussi, les théoriciens qui définissent la traduction à l'aide de la notion d'équivalence sont nombreux, pour ne citer que Nida qui propose que « la traduction consiste à produire dans la langue d'arrivée l'équivalent naturel le plus proche du message de la langue de départ, d'abord quant à la signification, puis quant au style » (Nida in Mounin 1976 : 278). Cette notion est ainsi partagée par les linguistes et les théoriciens de la traduction. En effet, les premiers l'associent à la langue en tant que système ou idiome (voir Pergnier 1993) ; les seconds placent l'équivalence sur le plan du discours et la perçoivent comme une interaction, voire comme la recherche d'une symétrie entre le traducteur et le texte. Or, la recherche de l'équivalence pose sans doute plus de difficultés en matière de traduction spécialisée, notamment dans le domaine du droit où les « traditions d'écriture, de cohérence et de logique diffèrent d'une langue à l'autre et, donc, d'un système à un autre » (Gémar 2006 : 79). En effet, en traduction juridique, l'équivalence des textes concerne aussi les effets juridiques, en plus de la forme. Le principe de l'équivalence des textes juridiques présente donc un intérêt réel et actuel.

Les questions relatives à la traduction spécialisée et à la langue juridique ayant déjà été traitées (entre autres, par Sourioux/Lerat 1975 ; Beaupré 1987 ; Lerat 1995 ; Gémar 2005), nous nous en tiendrons à des réflexions sur la traduction des textes juridiques. Dans cet article, nous analyserons quelques traductions juridiques pour aborder la question de la norme, liée inévitablement à la théorie de l'équivalence. Nous nous demanderons si le traducteur linguiste observe les mêmes normes et règles d'équivalence que le traducteur juriste. Rendent-ils à l'identique l'identité de sens, quelles que soient les divergences de structures (grammaticales, stylistiques ou lexicales) qui s'établissent entre les textes sources et cibles ? Puis, partant du modèle de Toury (1995) concernant l'activité traduisante, nous aborderons les écarts d'asymétrie entre traducteurs linguistes et traducteurs juristes.

\section{L'ÉQUIVALENCE OU LA QUÊTE DU FOND ET DE LA FORME}

La notion d'équivalence est un concept clé en traduction. En effet, depuis les premiers contacts entre groupes sociaux parlant des langues différentes, la traduction présente des homologies et des analogies (voir Hagège 1982: 9 et 1985 : 46-50).

Adresse de l'auteur : Filozofska fakulteta, Univerza v Ljubljani, Aškerčeva 2, 1000 Ljubljana, Slovénie. Courriel : sonia.vaupot@ff.uni-lj.si 
Cependant, cette notion demeure un phénomène confus et pragmatique, car elle met en jeu des données linguistiques complexes.

Jean Delisle (1993 : 29) assigne à la notion d'équivalence trois sens distincts. Tout d'abord, le sens général correspond à la relation d'identité entre deux unités de sens de langues différentes et ayant (presque) la même dénotation et la même connotation. Les équivalences sont toujours établies au niveau du discours à la suite d'une interprétation visant à dégager le sens du texte cible. Elles sont réalisées à la jonction de la connaissance de la langue et de la connaissance des réalités auxquelles renvoient au texte source, tous les paramètres de la communication étant pris en compte. Le deuxième sens de la notion d'équivalence, synonyme de traduction, précise le résultat de l'opération de traduction. Un troisième sens, plus restreint, traite l'équivalence comme un procédé de traduction consistant à rendre une expression figée de la langue source par une autre en langue cible qui, bien que différente, correspond à la même réalité.

Pour Marianne Lederer (1994 : 214), quelles que soient les divergences grammaticales ou lexicales, l'équivalence correspond à une identité de sens qui s'établit entre les textes et s'oppose à la correspondance qui unit éléments linguistiques, mots, syntagmes, figements ou formes syntaxiques. L'auteur (ibid. : 52-57) distingue aussi l'équivalence cognitive qui découle de la jonction du sémantisme du texte et des compléments notionnels apportés par l'auteur et l'équivalence affective car « l'équivalence est le résultat d'une méthode raisonnée (s'efforcer de voir et de ressentir) et de l'intuition (exprimer ce qu'on a vu et ressenti) ».

Partant de l'unité de traduction, Michel Ballard (1993 : 252-260) propose une typologie de l'équivalence. Il distingue : l'équivalence directe ou traduction littérale lorsque le segment d'arrivée est calqué sur le segment de départ; l'équivalence indirecte qui présente des aspects divers « mais commentables, analysables, déductibles »; l'équivalence idiomatique, souvent opaque à l'analyse et particulièrement caractéristique du langage parlé qui échappe au traducteur car il doit retrouver une formule dictée par l'usage du groupe et intervient surtout dans les dialogues ; l'équivalence culturelle ou l'intraduisibilité culturelle (voir aussi Catford 1965 : 99) dont « la forme brute et honnête » est l'emprunt, parfois assorti de notes, et relève d'une conception ethnocentrique de la traduction. À cela s'ajoute l'équivalence fonctionnelle qui intervient dans la traduction des jeux de mots, la poésie, les slogans publicitaires et dans les cas où la créativité et le fait d'envisager le sens comme la fonction du langage apparaissent. L'usage en traduction des types d'équivalences est, entre autres, caractérisé par les différents principes de « dominance, alternance, hiérarchisation, asymétrie, base et aboutissement de l'unité de traduction. »

Sechrest, Fay et Zaidi (1972 : 41-56) identifient quatre catégories principales de problèmes d'équivalence de traduction : l'équivalence de vocabulaire ou lexicale, l'équivalence idiomatique, l'équivalence grammaticale et syntaxique et l'équivalence d'expérience, c'est-à-dire que les mots traduits doivent se référer à des objets réels et des expériences réelles, lorsque ces expériences sont familières dans les deux langues et cultures. Ainsi, l'une des premières aspirations en traduction correspond à atteindre la parfaite comparabilité linguistique, c'est-à-dire, atteindre l'équivalence lexicale. Or, cette dernière est souvent trompeuse car apparente : même quand le signifiant ou le mot 
est traduit, le signifié ou la réalité ne correspondent pas dans leur totalité. L'adéquation n'est donc pas complète. En effet, un mot ou une phrase possèdent en général un équivalent mais il est nécessaire de savoir, de manière précise en traduction juridique, dans quelle mesure et jusqu'à quel point, le sens est équivalent dans les deux langues ou les deux cultures. Les difficultés de la traduction lorsque la réalité désignée reste la même résultent du fait que le découpage, souvent conceptuel, est différent d'une langue à $1^{\prime}$ autre. Or, ce fait, qui manifeste l'autonomie de la langue, est difficile à percevoir, accepter et rendre, car il se situe souvent à un autre niveau, plus implicite ou simplement différent. Aussi, pour Nida (1964 : 12-13), la traduction consiste à trouver l'équivalent naturel le plus proche, soit « The closes natural equivalent».

Enfin, Newmark (1988: 82-83) distingue l'équivalent culturel qui correspond à la traduction plus ou moins approximative d'un trait culturel équivalent de la langue source dans la langue cible : par exemple, le mot « Palais Bourbon » peut être traduit par « the French Westminster ». Mais, la traduction est limitée et imprécise. De nature fonctionnelle et parfois descriptive ( « la Cour de cassation » pourrait, par exemple, correspondre à « vrhovno sodišče »), leur usage est aussi réduit. L 'équivalent fonctionnel

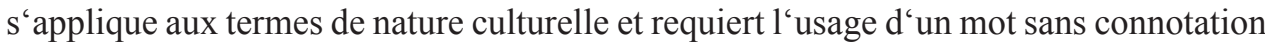
culturelle. Il s'agit de rendre neutre ou de généraliser le mot de la langue source. Ainsi, on peut traduire le terme " tribunal » par " sodišče ». Ce procédé, qui relève d'une analyse componentielle culturelle, consiste à déculturaliser ou généraliser un terme. $\mathrm{Ce}$ même type de procédé est également employé en cas de vide lexical, de nature technique, c'est-à-dire lorsqu'un terme technique ne possède pas d'équivalent.

Par conséquent, la question de l'équivalence, thème vaste, nous mène irrémédiab-

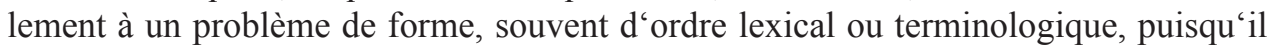
$s^{\prime}$ agit de différencier l'équivalence en tant qu'unité de traduction, stratégie et procédé (voir Gruntar Jermol 2009). D‘une part, le concept d'équivalence en traduction possède une portée trop importante pour être classé comme une sous-catégorie (voir, à ce propos, les procédés de traduction de Vinay/Darbelnet 1958). Pour sa part, Jakobson (1970 : 78-99) le remarquait déjà et Ladmiral $(1979$ : 20) était réservé à son sujet : « le concept ,d'équivalence' a une validité générale et il tend à désigner toute opération de traduction ». Le terme d'équivalence s'applique donc à la traduction d'un mot, d'un énoncé ou d'un texte et tend vers la reformulation du même sens, du même message, de la même réalité, de la même norme et de la même valeur, etc. Or, même traduit, un mot ne reflète pas tout à fait ces mêmes éléments. La traduction juridique en est un exemple flagrant.

D'autre part, le problème de l'équivalence rejoint notamment le problème de la synonymie (voir Jakobson 1970 : 78-100). En effet, si dans une même langue deux mots ne sont pas tout à fait équivalents comment le seraient-ils en traduction ? En traduction juridique, à l'instar de la traduction se pose le problème de l'interprétation du texte (une loi, par exemple) qui représente l'un des critères essentiels de l'équivalence recherchée par le traducteur.

D'une manière générale, il est donc possible de distinguer l'équivalence absolue ou totale (soit une adéquation complète), qui sert essentiellement à la traduction des 
termes spécialisés, et la plus courante, l'équivalence partielle (ou adéquation approximative). La première suppose que les équivalents sont substituables dans n'importe quel contexte. Ces cas sont rares et les équivalents absolus ne se rencontrent guère en dehors des nomenclatures techniques, scientifiques ou savantes. Si les vrais équivalents ou mots, ayant exactement le même sens, dans la langue source et la langue cible, se trouvent essentiellement dans le langage technique ou spécialisé, auquel cas l'équivalence en traduction serait limitée à ce type de traduction, les autres types relevant davantage de l'homologie de sens, de message et de code. La langue juridique penche à priori vers l'équivalence partielle. Ces éléments mettent en valeur le caractère arbitraire de la notion d'équivalence car, quels que soient la stratégie de traduction adoptée ou le type de texte, une partie du sens ou du message gardera un brin de son unicité par rapport à la langue source et la culture source.

Quant à Toury (1995:61), il rapproche la notion d'équivalence de celle de norme qui détermine le type et l'extension de l'équivalence dans un texte traduit. Il propose aussi la notion d'adéquation (adequacy) pour marquer la nature de la relation entre le texte source et le texte cible. Selon l'auteur (Toury 1980, 1995), le traducteur situe son texte dans une échelle entre « acceptabilité » et « adéquation », cette dernière se mesurant en fonction du respect aux normes du polysystème source. L'adéquation désigne ainsi l'un des deux pôles du continuum qui relève de la norme employée dans le processus de traduction, c'est-à-dire qu'une traduction est dite « adéquate» si le traducteur penche pour la langue source plutôt que pour la langue cible et respecte les normes littéraires. Pour la traduction juridique, l'équivalence de forme ne peut être remise en question, la lettre doit être respectée au même titre que l'équivalence de fond, le sens et l'interprétation sont tout aussi souhaitables. Or le traducteur d'un texte juridique peut-il atteindre ces deux objectifs? Quelles normes doit-il ou peut-il suivre?

\section{LES NORMES EN TRADUCTION JURIDIQUE}

Le Droit est constitué d'un ensemble de normes. Dans le droit national, les normes sont hiérarchisées. En droit international, il existe un principe général qui est celui de l'équivalence normative. La notion du Jus cogens est la norme impérative, au-dessus de toutes les autres: Jus cogens signifie en latin « le droit accepté par tous les gens qui vivent ensemble ». Il y a aussi l'idée que des gens vivant ensemble (cogens) produisent un droit commun. Ce concept est réapparu en 1969 avec la Convention de Vienne sur le Droit des Traités qui évoque ce concept romain dans les articles 53 et 54.

La langue repose aussi sur une convention ou une norme entre locuteurs. Le mot norme est ainsi polysémique. La norme, définie dans le cadre saussurien, avec la langue comme système "préexistant», lutte contre l'usage quotidien de la langue, la parole. Cette norme saussurienne est désintégrée en norme implicite, explicite et fonctionnelle. D'autre part, la norme est arbitraire linguistiquement. Une langue possède des normes acceptées par un groupe ou une collectivité.

Dans le domaine de la traduction, selon Toury (1980), le concept de norme diffère de l'approche normative. Les normes sont descriptives, elles ne désignent ni les règles 
ni les contraintes imposées aux traducteurs ; elles ne peuvent pas évaluer la traduction, mais reflètent les conditions de réalisation et de réception des traductions dans un contexte spécifique. Dans son ouvrage de 1995, Toury présente la théorie des normes de la traduction littéraire. Il constate que le traducteur possède un rôle social qui dépasse le simple transfert linguistique. L'auteur définit, entre autres, 3 types de normes : les normes initiales (le traducteur adhère aux normes du texte source ou cible) ; les normes préliminaires (le traducteur a le choix quant au type de texte, à l'origine des auteurs, aux sujets...) et les normes opérationnelles (le traducteur prend des décisions en matière de segmentation du texte ou reformulation et tient compte des normes linguistiques et textuelles).

Les textes juridiques sont des textes porteurs de normes juridiques (les lois, par exemple). En sus des (non) concordances juridiques apparaissent les contraintes linguistiques : la traduction doit adhérer au style juridique et être lisible par le destinataire. Aussi, on peut se demander si, dans le domaine juridique, une traduction doit ou peut être identique à l'original, ou encore fidèle en tout point. La norme juridique conférant au texte toute sa spécificité, la traduction doit-elle adhérer, complètement ou partiellement, aux différentes normes (linguistiques, terminologiques, institutionnelles, etc.) ? L'équivalence des textes dans chaque système peut-elle être parfaite ou bien n'est-elle que forcée ? Enfin, les traductions effectuées par des linguistes diffèrent-elles, dans la forme ou le fond, de celles des juristes?

\section{CARACTÉRISTIQUES SYNTAXIQUES}

Le caractère normatif et parfois contraignant de la traduction juridique n'est plus à prouver. Ce domaine exige une traduction ou les mots sont parfois univoques et ne répondent pas toujours à l'activité normative. Certaines spécificités du texte juridique ont déjà été évoquées dans un précédent article (voir Vaupot 2009). Ainsi, à l'instar de la terminologie et du lexique, la syntaxe peut présenter des écueils.

Les caractéristiques linguistiques des textes normatifs s'expliquent notamment par le fait que les conditions particulières entraînent des spécificités, souvent textuelles. En effet, la langue du droit présente quant à elle des structures syntaxiques préférentielles. La compétence rédactionnelle en langue cible permet au traducteur de respecter les particularités de structuration spécifiques à chaque langue en assurant la qualité d'une traduction et l'équivalence du texte. Toutefois, même si la langue du droit répond aux mêmes normes et règles syntaxiques que la langue courante, des spécificités apparaissent, entre autres, dans l'ordre des mots. En comparant les propriétés des langues françaises et slovènes, on note que le français est une langue plutôt analytique et le slovène plutôt synthétique. L'ordre des mots en slovène est en général plus libre et ne correspond pas systématiquement à la structure de la phrase française la plus simple qui comprend un sujet, un verbe et un complément. Le slovène possède un choix de possibilités relativement vaste et s'articule selon le principe d'actualité, de sorte qu'un énoncé peut être nuancé par l'ordre utilisé. Ainsi, l'élément le plus important dans la phrase est celui qui est le plus actuel. Lorsque l'ordre des mots met en évidence un 
élément attendu et objectif, cet ordre est réparti selon le concept de perspective de sens qui s'appuie sur les notions suivantes :

- le point de départ (izhodišče) : l'élément connu est placé en début de phrase : oseba mora prevzeti odgovornost;

- le passage ou la transition (prehod), soit le noyau verbal : vedno mora oseba prevzeti odgovornost ;

- le noyau (jedro) : l'élément inconnu, nouveau ou mis en relief est ajouté en fin de phrase : Nemiri in vasi se je ogibal.

En traduction, la syntaxe française ne pourra correspondre à la lettre à celle du slovène. En effet, le français pourra placer l'élément (verbe, attribut ou complément) mis en relief en tête de phrase, tandis que le slovène l'ajoutera plutôt à la fin pour attirer l'attention du lecteur (fonction appellative), parfois aussi en début de phrase (fonction expressive). En français, le fait de rejeter l'élément en fin de phrase consiste à le souligner plus ou moins fortement.

Comparé à d'autres types de textes, le texte juridique, français ou slovène, est de nature normative et comprend des spécificités syntaxiques. Les formes syntaxiques classiques, en français, sont ainsi soumises à des transformations (voir Sourioux/Lerat 1975) dont nous ne citerons que quelques exemples : le ton est neutre ; on emploie le présent de l'indicatif notamment dans la loi et le règlement, le masculin, les constructions passives inachevées sans complément d'agent, les constructions pronominales de sens passif, les constructions impersonnelles (il convient) ; on note l'absence du pronom indéfini « on », mais aussi la présence du sujet indéfini (quiconque, nul), des marques négatives (il n'y a pas de mariage...), des constructions restrictives (nul ne doit), les marques démonstratives (ci-après) ; le souci de précision étant permanent, les juristes ont recours aux adjectifs comme « ledit» ou aux relatifs « lequel»; la redondance caractérise aussi les textes juridiques français.

Aussi, on se demandera quelles sont les normes prises en considération par le traducteur slovène face au texte juridique. L'approche traductionnelle est-elle plutôt sourcière ou cibliste ? L'ordre des phrases est-il calqué sur le texte source ou, à l'opposé, la syntaxe et le style sont-ils adaptés aux lois et institutions de l'Autre ? Enfin, le style et les stratégies du traducteur linguiste se différencient-ils du traducteur juriste?

\section{STRATÉGIES ET PERSPECTIVES DE TRADUCTION}

En réponse à cette problématique, nous avons analysé un corpus bilingue. Notre premier corpus, tiré du Journal officiel slovène (Uradni list RS, 108/04), plus particulièrement du site institutionnel Evrokorpus, comporte plusieurs textes traduits en slovène. En matière de traduction, la langue juridique slovène est respectée. Mais, dans le texte français, le mois de «novembre » étant écrit avec une majuscule, on peut se demander si l'original est bien français. En outre, tout au long du texte, l'ordre des mots, dans les deux langues, est presque identique. Par exemple : 
(a) Le Comité de Gestion se réunira selon les besoins et au moins une fois par an, alternativement en Slovénie et en France, sauf accord entre le MVZT et le CEA, et la date de la réunion, l'ordre du jour et les détails pratiques seront définis d'un commun accord par les coordinateurs, étant précisé que l'organisateur de chaque réunion sera le coordinateur de la Partie dans le pays de laquelle la réunion est organisée.

(b) Upravni odbor se sestaja po potrebi in vsaj enkrat na leto izmenično v Sloveniji in Franciji, razen če se MVZT in CEA ne dogovorita drugače; datum sestanka, dnevni red in praktične podrobnosti sporazumno določita koordinatorja, s tem da je organizator vsakega sestanka obenem koordinator pogodbenika v državi, $v$ kateri se sestanek organizira.

Toutefois, la syntaxe slovène se démarque parfois de l'original français en employant certains procédés de modulation ou de transposition (pris au sens de Vinay/ Darbelnet 1958) :

- La nominalisation : de modifier les règles et procédures concernant les expositions internationales/sprememba pravil in postopkov v zvezi z mednarodnimi razstavami;

- La chute de certains éléments : Le présent Protocole est ouvert à la signature des Parties à la Convention de 1928 à Paris du 30 Novembre 1972 au 29 Novembre 1973 et restera ouvert après cette dernière date pour l'adhésion de ces mêmes Parties/Ta protokol je na voljo za podpis pogodbenicam konvencije iz leta $1928 \mathrm{v}$ Parizu od 30. novembra 1972 do 29. novembra 1973 in po tem datumu ostane na voljo za njihov pristop.

- La verbalisation : Leur durée ne peut être inférieure à six semaines, ni supérieure à six mois/Trajati ne smejo manj kot šest tednov in več kot šest mesecev.

On peut en conclure que l'approche traductionnelle choisie par le traducteur linguiste est surtout sourcière. L'ordre des phrases slovènes semble calqué sur le texte source, alors qu'on aurait pu prévoir plus de divergences syntaxiques.

Le second corpus est tiré du Journal officiel de l'Union européenne (C 115/210), datant du 09/05/2008, version bilingue. Même si l'anglais constitue la langue dominante, notamment en matière de traduction de l'acquis communautaire, le style du corpus nous permet de penser que le texte original est bien français. Contrairement aux présupposés, dans les exemples suivants, nous notons que le traducteur (juriste) a opéré des changements plus importants : il a adapté la phrase en ajoutant «l'article 160 » à la version slovène (d). Il a aussi attribué des chiffres différents dans l'exemple (f) : on passe de 14 et 13 (version française) à 13 et 12 juges (version slovène).

(c) DÉSIRANT fixer le statut de la Cour de justice de l'Union européenne prévu à l'article 281 du traité sur le fonctionnement de l'Union européenne,

(d) ZŽELJO določiti statut Sodišča, predviden v členu 281 Pogodbe o delovanju Evropske unije in členu 160 Pogodbe o ustanovitvi Evropske skupnosti za atomsko energijo,

(e) Le renouvellement partiel des juges, qui a lieu tous les trois ans, porte alternativement sur quatorze et treize juges. 
(f) Ko se vsaka tri leta izvede delna zamenjava sodnikov, se izmenično zamenja trinajst in dvanajst sodnikov.

Dans la traduction, la langue juridique slovène est respectée, mais certains termes (g) sont parfois précisés et explicités, sans doute pour plus de clarté et une meilleure adéquation $(\mathrm{h})$ :

(g) "Le Tribunal est formé de vingt-sept juges. "

(h) "Sodišče prve stopnje ima sedemindvajset sodnikov. "

D'autres vont résumer et impliciter des éléments, comme l'indiquent les exemples suivants (j) et (1) :

(i) Tout juge doit, avant d'entrer en fonctions, devant la Cour de justice siégeant en séance publique, prêter serment d'exercer ses fonctions en pleine impartialité et en toute conscience et de ne rien divulguer du secret des délibérations.

(j) Pred nastopom funkcije vsak sodnik pred Sodiščem in javno priseže, da bo svoje naloge opravljal nepristransko in vestno ter da bo varoval tajnost posvetovanj Sodišča.

(k) Un pourvoi peut être formé devant la Cour de justice contre les décisions du Tribunal rejetant une demande d'intervention, dans un délai de deux semaines à compter de la notification de la décision de rejet, par toute personne dont la demande a été rejetée.

(1) Vsakdo, čigar vlogo za pridružitev postopku je Splošno sodišče zavrnilo, se lahko $v$ dveh mesecih po uradnem obvestilu o odločitvi o zavrnitvi vloge pritoži na Sodišče.

Dans ces corpus, les débuts de phrases sont grammaticalement identiques (essentiellement nominales) et la traduction calque l'ordre des mots : les traducteurs emploient ainsi des stratégies syntaxico-grammaticales (voir Chesterman 1997). Mais, le traducteur juriste vise plutôt une perspective cibliste, en utilisant notamment des stratégies de traduction pragmatiques (ibid.), tandis que le traducteur linguiste tend vers une perspective sourcière et opère surtout des changements linguistiques, ayant ainsi recours à des stratégies sémantiques (ibid.).

\section{CONCLUSION}

Si nous appliquons le modèle de Toury (1995) à l'activité traduisante en Slovénie et, plus particulièrement, aux traductions des textes juridiques vers le slovène, on remarque d'une manière générale que les traducteurs linguistes, dans le cadre institutionnel, suivent de près les normes en tant que cadre imposé et sont peu amenés à tenir compte des conventions prises dans le sens de préférences personnelles. Ils suivent surtout les règles constitutives ou règles préexistantes à l'activité traductive et moins les règles régulatrices ou règles internes au domaine traduit. Les normes professionnelles et institutionnelles slovènes (notamment en traduction juridique) prônent la traduction proche de l'original, donc une approche sourcière, dans la 
perspective des normes initiales. Le traducteur linguiste semble ainsi adhérer à la langue et culture source.

Dans le second cas, le traducteur juriste se soumet aux normes linguistiques de la culture cible pour que le texte cible puisse trouver sa place dans le système de la culture d'arrivée. Son approche est donc cibliste. Les normes sont concurrentes puisqu'il existe plusieurs règles en compétition. Elles sont également préliminaires car la politique de traduction est axée sur le choix du texte source. Elles sont opérationnelles ou opératoires lorsqu'elles orientent les décisions traductives et offrent une traduction différente selon la formation initiale du traducteur, le statut, l'expérience et le public cible ; elles dirigent aussi les choix du traducteur pendant le processus de traduction.

Les corpus analysés ne sont certes que des échantillons, mais ils révèlent différentes approches et stratégies ainsi qu'une dualité et une orientation différente dans les perspectives de traduction puisque les linguistes semblent préférer la tendance sourcière contrairement aux spécialistes qui tendent vers l'approche cibliste. Une étude plus large permettrait sans doute de nuancer nos hypothèses. Enfin, en matière de traduction juridique, l'identité de sens est rendue à l'identique, les linguistes comme les juristes respectent les normes et tendent en général vers l'équivalence partielle.

Cette problématique soulève encore d'autres questions, notamment celles de la qualité des traductions, des difficultés de la langue de la traduction, de la formation antérieure et actuelle des traducteurs-linguistes et traducteurs-juristes, ou encore de la façon dont le public perçoit le message dans la langue cible. Ces questions demeurent en suspens.

\section{Bibliographie}

\section{Sources primaires}

Protokol (št. 3) o statutu sodišča evropske unije. Juillet 2013. http://eur-lex.europa.eu.

Uradni list RS, št. 113/03 - uradno prečiščeno besedilo. Juillet 2013. http://www.evroterm.gov.si.

\section{Références}

BEAUPRÉ, Michael (1987) « La traduction juridique : introduction. » Les Cahiers du Droit 28/4, 735-745.

BALLARD, Michel (1993) La traduction à l'université - Recherches et propositions didactiques. Lille : Presses Universitaires de Lille.

CATFORD, John C. (1965) A Linguistic Theory of Translation. London : Oxford University Press.

CHESTERMAN, Andrew (1997) Memes of translation: The spread of ideas in translation theory. Amsterdam : Benjamins.

DELISLE, Jean (1993) La traduction raisonnée-Manuel d'initiation à la traduction professionnelle de l'anglais vers le français. Ottawa : Les Presses Universitaires d'Ottawa. 
GEMAR, Jean-Claude (2005) «Interpréter le sens, produire l'équivalence : obligations de résultat du traducteur. » In : F. Israël/M. Lederer (eds), La Théorie interprétative de la traduction - Convergences, mises en perspective (Vol. II). Paris-Caen, Lettres Modernes Minard. Paris : Cahiers Champollion 7, 212-228.

GEMAR, Jean-Claude (2006) «Traduction spécialisée et droit. Langage du droit, styles et sens. » Insights into Specialized Translation 46, 79-106.

GRUNTAR JERMOL, Ada (2009) « Rechtstexte übersetzen - leicht gemacht? Oder : Wie schnell kann man sich beim Übersetzen juristischer Texte verlaufen. » Terminology 15/2, 214-231.

HAGEGE, Claude (1982) La structure des langues. Paris : Presses Universitaires de France.

HAGEGE, Claude (1985) L'Homme de paroles. Paris : Fayard.

JAKOBSON, Roman (1970) Essais de linguistique générale. Paris : Minuit.

LADMIRAL, Jean René (1979) Traduire : théorèmes pour la traduction. Paris : Payot.

LEDERER, Marianne (1994) La traduction aujourd'hui - Le modèle interprétatif. Paris : Hachette.

LERAT, Pierre (1995) Les langues spécialisées. Paris : Presses Universitaires de France.

MOUNIN, Georges (1976) Les problèmes théoriques de la traduction. Paris : Gallimard.

NEWMARK, Peter (1988) A Textbook of Translation. Hemel Hempstead : Prentice Hall.

NIDA, Eugène A. (1964) Toward a science of translating. Leiden : E. J. Brill.

PERGNIER, Maurice (1993) Les fondements socio-linguistiques de la traduction. Lille : Presses Universitaires de Lille.

SECHREST, Lee/Todd L. FAY/Syed M. HAFEEZ ZAIDI (1972) « Problems of Translation in Cross-Cultural Research. " Journal of Cross-Cultural Psychology 3/1, $41-56$.

SOURIOUX, Jean-Louis/Pierre LERAT (1975) Le langage du droit. Paris : Presses universitaires de France.

TOURY, Gideon (1980) Descriptive Translation Studies and beyond. Amsterdam/Philadelphia : John Benjamins Publishing.

TOURY, Gideon (1995) Descriptive Translation Studies - and Beyond. Amsterdam/ Philadelphia : John Benjamins Publishing.

VAUPOT, Sonia (2009) «L'enseignement de la terminologie juridique française à un public slovène. » Terminology 15/1, 119-143.

VINAY, Jean-Paul/Jean DARBELNET (1958) Stylistique comparée du français et de l'anglais. Paris : Didier. 


\section{Résumé \\ ÉQUIVALENCE ET NORMES EN TRADUCTION JURIDIQUE}

La traduction juridique est un processus complexe au cours duquel le traducteur doit prendre une série de décisions. En effet, il doit traduire les mots et le texte tout en laissant les normes inchangées. S'il s'agit de traduire des normes juridiques, la traduction consiste à faire en sorte que le texte produise dans la langue cible les mêmes effets que dans la langue source. Ainsi, dans le présent article, nous tenterons de préciser en quoi la notion de norme intéresse la traduction juridique. Par ailleurs, la notion de norme implique inévitablement une théorie de l'équivalence. En effet, ce type de traduction exige des connaissances particulières dans le domaine juridique dans la langue source comme dans la langue cible. Aussi, partant d'un corpus, nous nous demanderons si le traducteur linguiste observe les mêmes règles d'équivalence que le traducteur juriste. Rendent-ils à l'identique l'identité de sens quelles que soient les divergences de structures (grammaticales, stylistiques ou lexicales) qui s'établissent entre les textes sources et cibles ? Partant du modèle de Toury concernant l'activité traduisante, nous l'appliquerons aux traductions des textes juridiques, mettant ainsi en évidence les similitudes ou divergences entre traducteurs linguistes et traducteurs juristes.

Mots-clés : Traduction, équivalence, normes, droit, français/slovène.

\section{Povzetek \\ O EVIVALENCI IN NORMI PRI PREVAJANJU PRAVNIH BESEDIL}

Prevajanje pravnih besedil je kompleksen proces, $\mathrm{v}$ katerem mora prevajalec sprejeti vrsto odločitev. Tako pri prevodu posameznih besed oz. terminov kot tudi celotnega besedila naj bi prevajalske norme ostale nespremenjene. Ko gre za prevajanje pravnih norm, naj bi ciljno besedilo imelo enak učinek kot izvirnik.

V članku skušamo določiti povezavo med normo in prevajanjem pravnih besedil. Pojem norme neogibno vključuje tudi teorijo ekvivalence. Poleg tega je pri pravnem prevajanju nujno potrebno ustrezno strokovno znanje tako izhodiščnega kot tudi ciljnega pravnega jezika in sistema. Na podlagi raziskav izbranega korpusa skušamo ugotoviti, ali prevajalec-jezikoslovec upošteva ista pravila ekvivalence kot prevajalec-pravnik in ali oba $\mathrm{v}$ besedilu ohranita enak pomen, ne glede na razlike v strukturah (slovničnih, slogovnih oz. leksikalnih) med izvirnikom in prevodom. Za prevajanje pravnih besedil uporabljamo Touryjev $(1980,1995)$ prevajalski model, pri čemer se osredotočamo predvsem na podobnosti in razlike pri prevodih prevajalcev-jezikoslovcev in prevajalcev-pravnikov.

Ključne besede: prevajanje, ekvivalenca, norme, pravo, francosko/slovensko. 